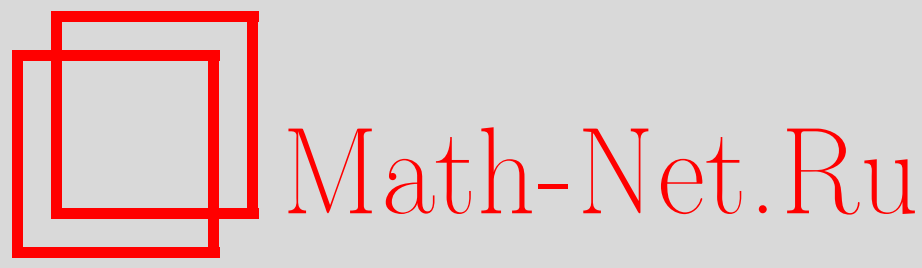

Л. Н. Липатов, Дважды-логарифмическая асимптотика для амплитуд рассеяния в гравитации и супергравитации, ТМФ, 2013, том 175, номер 3, 408-418

DOI: https://doi.org/10.4213/tmf8489

Использование Общероссийского математического портала Math-Net.Ru подразумевает, что вы прочитали и согласны с пользовательским соглашением http://www.mathnet.ru/rus/agreement

Параметры загрузки:

IP: 3.85 .73 .92

26 апреля 2023 г., $10: 10: 28$

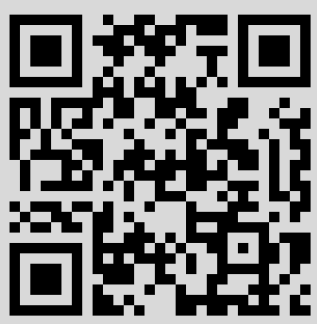




\title{
ФИЗИКА
}

Том 175, № 3

июнь, 2013

(C) 2013 г.

\author{
Л. Н. Липатов*
}

\section{ДВАЖДЫ-ЛОГАРИФМИЧЕСКАЯ АСИМПТОТИКА ДЛЯ АМПЛИТУД РАССЕЯНИЯ В ГРАВИТАЦИИ И СУПЕРГРАВИТАЦИИ}

\begin{abstract}
Дается обзор подхода Балицкого-Фадина-Кураева-Липатова к рассеянию при высоких энергиях в квантовой хромодинамике и в суперсимметричных калибровочных теориях. В пределе большого числа цветов уравнения для составных глюонных состояний в $t$-канале рассеяния имеют замечательные математические свойства, в том числе инвариантность относительно преобразований Мёбиуса, голоморфную факторизацию, дуальную симметрию и интегрируемость. Теория реджезованных глюонных взаимодействий формулируется в виде калибровочно-инвариантного эффективного действия, локального по быстротам частиц. В максимально расширенной $N=4$ суперсимметрии померон оказывается дуальным реджезованному гравитону, живущему в десятимерном пространстве анти-де Ситтера. В результате возникает необходимость переформулировать подход Грибова к померонным взаимодействиям в виде общековариантной эффективной теории поля для реджезованных гравитонов. Построено соответствующее эффективное действие, позволяющее вычислять траектории Редже гравитона и его взаимодействия. Произведено суммирование дважды-логарифмических вкладов для амплитуд с квантовыми числами гравитона в $t$-канале рассеяния в гравитации Эйнштейна-Гильберта и в ее суперсимметричных обобщениях. С ростом ранга $N$ супергравитации дважды-логарифмические амплитуды быстро убывают по сравнению с борновскими вкладами.
\end{abstract}

Ключевые слова: квантовая гравитация, высокоэнергетическая асимптотика, поведение амплитуд типа Редже, приближение двойных логарифмов.

DOI: $10.4213 / \operatorname{tmf} 8489$

\section{1. АМПЛИТУДЫ РАССЕЯНИЯ В КХД ПРИ ВЫСОКИХ ЭНЕРГИЯХ}

Борновская амплитуда рассеяния в КХД при высоких энергиях $s \gg t$ имеет простой факторизованный вид

$$
M_{A B \text { Born }}^{A^{\prime} B^{\prime}}=2 s g T_{A^{\prime} A^{\prime}}^{c} \delta_{\lambda_{A^{\prime}} \lambda_{A}} \frac{1}{t} g T_{B^{\prime} B}^{c} \delta_{\lambda_{B^{\prime}} \lambda_{B}}, \quad \lambda_{i}= \pm 1,
$$

*Петербургский институт ядерной физики, Гатчина, Ленинградская обл., Россия. E-mail: lipatov@thd.pnpi.spb.ru 
отвечающий сохранению спиральности $\lambda_{i}=\lambda_{i^{\prime}}$ для каждой из сталкивающихся частиц. В ведущем логарифмическом приближении (ВЛП) глюон в перекрестном канале рассеяния становится реджезованным [1]:

$$
M(s, t)=M_{\left.\right|_{\text {Born }}} s^{\omega(t)}, \quad \alpha_{s} \ln s \sim 1, \quad \alpha_{s}=\frac{g^{2}}{4 \pi} \ll 1 .
$$

Траектория глюона в ВЛП имеет вид

$$
\omega\left(-|q|^{2}\right)=-\frac{\alpha_{s} N_{\mathrm{c}}}{4 \pi^{2}} \int d^{2} k \frac{|q|^{2}}{|k|^{2}|q-k|^{2}} \approx-\frac{\alpha_{s} N_{\mathrm{c}}}{2 \pi} \ln \frac{\left|q^{2}\right|}{\lambda^{2}}
$$

Инфракрасная расходимость при $\lambda \rightarrow 0$ сокращается в полном сечении рассеяния. Парциальная волна в $t$-канале с октетными квантовыми числами удовлетворяет уравнению бутстрапа

$$
\omega f=1+\left(\omega\left(-|k|^{2}\right)+\omega\left(-|q-k|^{2}\right)+\widehat{K}_{8}\right) f, \quad f=\frac{1}{\omega-\omega\left(-|q|^{2}\right)},
$$

обязанному своим происхождением тому обстоятельству, что реджезованный глюон представляет собой связанное состояние двух реджезованных глюонов.

Схожим образом амплитуда рождения $n$ частиц в высокоэнергетической кинематике $s \gg s_{i} \gg\left|t_{i}\right|$ имеет факторизованный вид [1]:

$$
M_{2 \rightarrow 2+n}^{\mathrm{BFKL}} \sim \frac{s_{1}^{\omega_{1}}}{\left|q_{1}\right|^{2}} g T_{c_{2} c_{1}}^{d_{1}} C\left(q_{2}, q_{1}\right) \frac{s_{2}^{\omega_{2}}}{\left|q_{2}\right|^{2}} \ldots g T_{c_{n+1} c_{n}}^{d_{n}} C\left(q_{n+1}, q_{n}\right) \frac{s_{n+1}^{\omega_{n+1}}}{\left|q_{n+1}\right|^{2}},
$$

где $\omega_{r}$ - траектории полюсов Редже в парциальных волнах $t_{i}$-каналов рассеяния, а $C\left(q_{r}, q_{r-1}\right)$ суть эффективные вершины для рождения глюона с определенной спиральностью:

$$
\omega_{r}=-\frac{\alpha_{s} N_{\mathrm{c}}}{2 \pi} \ln \frac{\left|q_{r}^{2}\right|}{\lambda^{2}}, \quad C\left(q_{2}, q_{1}\right)=\frac{q_{2} q_{1}^{*}}{q_{2}^{*}-q_{1}^{*}}, \quad \sigma_{t}=\sum_{n} \int d \Gamma_{n}\left|M_{2 \rightarrow 2+n}\right|^{2} .
$$

В силу факторизованного вида амплитуды рождения полное сечение рассеяния удовлетворяет уравнению Бете-Солпитера. Уравнение для соответствующей амплитуды упругого рассеяния с цветовыми синглетными квантовыми числами в перекрестном канале было много лет назад получено Балицким, Фадиным, Кураевым и Липатовым (БФКЛ) [1]. Поправки к этому уравнению в следующем за ведущим порядке получены сравнительно недавно [2]. Однородное уравнение БФКЛ в ВЛП совпадает с уравнением Шредингера для волновой функции померона, состоящего из двух реджезованных глюонов:

$$
E \Psi\left(\vec{\rho}_{1}, \vec{\rho}_{2}\right)=H_{12} \Psi\left(\vec{\rho}_{1}, \vec{\rho}_{2}\right), \quad \sigma_{t} \sim s^{\Delta}, \quad \Delta=-\frac{\alpha_{s} N_{\mathrm{c}}}{2 \pi} E_{0} .
$$


Гамильтониан, отвечающий этому уравнению, можно представить в следующем операторном виде [3]:

$$
H_{12}=\frac{1}{p_{1} p_{2}^{*}}\left(\ln \left|\rho_{12}\right|^{2}\right) p_{1} p_{2}^{*}+\frac{1}{p_{1}^{*} p_{2}}\left(\ln \left|\rho_{12}\right|^{2}\right) p_{1}^{*} p_{2}+\ln \left|p_{1} p_{2}\right|^{2}-4 \psi(1),
$$

где введены комплексные обозначения $\rho_{12}=\rho_{1}-\rho_{2}$ и $\rho_{r}=x_{r}+i y_{r}, r=1,2$.

Оператор $H_{12}$ инвариантен относительно преобразований Мёбиуса [4]

$$
\rho_{k} \rightarrow \frac{a \rho_{k}+b}{c \rho_{k}+d}, \quad m=\gamma+\frac{n}{2}, \quad \tilde{m}=\gamma-\frac{n}{2}, \quad \gamma=\frac{1}{2}+i \nu,
$$

где $\gamma$ - аномальная размерность оператора твиста 2 , а $n$ - целочисленный конформный спин. Собственные значения оператора $H_{12}$ имеют вид

$$
E=\epsilon_{m}+\epsilon_{\widetilde{m}}, \quad \epsilon_{m}=\psi(m)+\psi(1-m)-2 \psi(1)
$$

что позволяет вычислить померонный интерсепт

$$
\Delta=\frac{g^{2} N_{\mathrm{c}}}{\pi^{2}} \ln 2>0
$$

В результате в ВЛП получим нарушение ограничения Фруассара $\sigma_{t}<c \ln ^{2} s$ для полного сечения рассеяния.

Для восстановления унитарности в $s$-канале рассеяния, потерянной в ВЛП, следует учесть вклад диаграмм с произвольным числом глюонов в перекрестном канале рассеяния. Уравнения для бесцветных составных состояний $n$ реджезованных глюонов были получены Бартельсом, Квичинским и Прашаловичем (БКП). В пределе больших $N_{\text {с }}$ соответствующий гамильтониан обладает свойством голоморфной сепарабельности [5]:

$$
H=\frac{1}{2}\left(h+h^{*}\right), \quad h=\sum_{k=1}^{n}\left(\ln \left(p_{k} p_{k+1}\right)+\frac{1}{p_{k}} \ln \left(\rho_{k, k+1} \rho_{k-1, k}\right) p_{k}-2 \psi(1)\right) .
$$

Оно влечет свойство голоморфной факторизации собственных функций:

$$
\Psi\left(\vec{\rho}_{1}, \vec{\rho}_{2}, \ldots, \vec{\rho}_{n}\right)=\sum_{r, s} a_{r, s} \Psi_{r}\left(\rho_{1}, \ldots, \rho_{n}\right) \Psi_{s}\left(\rho_{1}^{*}, \ldots, \rho_{n}^{*}\right)
$$

Голоморфная факторизация в двумерных конформных теориях поля есть следствие инвариантности относительно бесконечномерной группы Вирасоро [6]. Уравнение БКП также инвариантно относительно преобразования дуальности [7]

$$
\rho_{r} \rightarrow p_{r} \rightarrow \rho_{r+1}
$$

которое сходно по природе с симметрией гармонического осциллятора.

Голоморфное уравнение БКП интегрируемо [3], [8]. В самом деле, можно ввести матрицу монодромии

$$
t(u)=L_{1} L_{2} \ldots L_{n}=\left(\begin{array}{cc}
A(u) & B(u) \\
C(u) & D(u)
\end{array}\right), \quad L_{k}=\left(\begin{array}{cc}
u+\rho_{k} p_{k} & p_{k} \\
-\rho_{k}^{2} p_{k} & u-\rho_{k} p_{k}
\end{array}\right),
$$


удовлетворяющую уравнению Янга-Бакстера [8], [9]

$$
t_{r_{1}^{\prime}}^{s_{1}}(u) t_{r_{2}^{\prime}}^{s_{2}}(v) l_{r_{1} r_{2}}^{r_{1}^{\prime} r_{2}^{\prime}}(v-u)=l_{s_{1}^{\prime} s_{2}^{\prime}}^{s_{1} s_{2}}(v-u) t_{r_{2}}^{s_{2}^{\prime}}(v) t_{r_{1}}^{s_{1}^{\prime}}(u), \quad \hat{l}=u \hat{1}+i \widehat{P}
$$

След матрицы монодромии представляет собой трансфер-матрицу, которая является производящей функцией интегралов движения. Оказывается, что гамильтониан БКП есть локальный гамильтониан интегрируемой спиновой цепочки Гейзенберга, спины которой совпадают с генераторами группы Мёбиуса [8], [10].

\section{2. ВЫСОКОЭНЕРГЕТИЧЕСКОЕ ЭФФЕКТИВНОЕ ДЕЙСТВИЕ КХД}

Чтобы вычислить траектории и все возможные взаимодействия реджезованных глюонов, можно использовать эффективное действие [11], описывающее взаимодействие этих глюонов с обычными кварками и глюонами в форме, локальной в пространстве быстрот

$$
y=\frac{1}{2} \ln \frac{\epsilon_{k}+|k|}{\epsilon_{k}-|k|}, \quad\left|y-y_{0}\right|<\eta, \quad \eta \ll \ln s .
$$

Для обычных и реджезованных глюонов введем поля

$$
v_{\mu}(x)=-i T^{a} v_{\mu}^{a}(x), \quad A_{ \pm}(x)=-i T^{a} A_{ \pm}^{a}(x), \quad \delta A_{ \pm}(x)=0
$$

Калибровочно-инвариантные поля $A_{ \pm}$описывают рождение и уничтожение реджезованных глюонов в перекрестном канале рассеяния. Они удовлетворяют кинематическим связям $\partial_{\mp} A_{ \pm}=0$. Выпишем эффективное действие в терминах этих полей:

$$
S=\int d^{4} x\left(L_{\mathrm{QCD}}+\operatorname{Tr}\left(V_{+} \partial_{\mu}^{2} A_{-}+V_{-} \partial_{\mu}^{2} A_{+}\right)\right)
$$

где

$$
V_{+}=-\frac{1}{g} \partial_{+} \operatorname{Pexp}\left(-g \int_{-\infty}^{x^{+}} d\left(x^{\prime}\right)^{+} v_{+}\left(x^{\prime}\right)\right)=v_{+}-g v_{+} \frac{1}{\partial_{+}} v_{+}+\cdots .
$$

Известны правила Фейнмана для соответствующих диаграмм эффективной теории [12].

В качестве примера применения этого эффективного действия рассмотрим выражение для высокоэнергетической амплитуды рождения двух глюонов при глюонглюонных столкновениях в $N=4$ суперсимметричной теории. Это выражение было получено в следующем за ведущим логарифмическом приближении для амплитуд с максимальным нарушением спиральности при больших $N_{\text {c }}$. Соответствующая поправочная функция $R$ для анзаца Берна-Диксона-Смирнова [13] имеет вид [14]

$$
R e^{i \pi \delta}=\cos \pi \omega_{a b}+i \frac{a}{2} \sum_{n=-\infty}^{\infty}(-1)^{n} e^{i \phi n} \int_{-\infty}^{\infty} d \nu \frac{|w|^{2 i \nu}}{\nu^{2}+n^{2} / 4} \Phi(\nu, n)\left(\frac{-1}{\sqrt{u_{2} u_{3}}}\right)^{\omega(\nu, n)}
$$


и зависит от трех ангармонических отношений

$$
u_{1}=\frac{s s_{2}}{s_{012} s_{123}}, \quad u_{2}=\frac{s_{1} t_{3}}{s_{012} t_{2}}, \quad u_{2}=\frac{s_{3} t_{1}}{s_{123} t_{2}} .
$$

В соотношении (7)

$$
w=\sqrt{\frac{u_{2}}{u_{3}}} e^{i \phi}, \quad \cos \phi=\frac{1-u_{1}-u_{2}-u_{3}}{2 \sqrt{u_{2} u_{3}}}
$$

и используются обозначения

$\delta=\frac{\gamma_{\mathrm{K}}}{8} \ln \frac{|w|^{2}}{|1+w|^{4}}, \quad \omega_{a b}=\frac{\gamma_{\mathrm{K}}}{8} \ln |w|^{2}, \quad \Phi(\nu, n)=1-a\left(\frac{E_{\nu n}^{2}}{2}+\frac{3}{8} \frac{n^{2}}{\left(\nu^{2}+n^{2} / 4\right)^{2}}+\zeta(2)\right)$,

где $\gamma_{\mathrm{K}}$ - аномальная размерность каспа (угла на контуре).

Приведем выражение для собственного значения уравнения БФКЛ в присоединенном представлении:

$\omega(\nu, n)=-a E_{\nu, n}-a^{2}\left(\epsilon_{\nu n}^{\mathrm{FL}}+3 \zeta(3)\right), \quad E_{\nu n}=-\frac{|n| / 2}{\nu^{2}+n^{2} / 4}+2 \operatorname{Re} \psi\left(1+i \nu+\frac{|n|}{2}\right)-2 \psi(1)$,

где первая поправка имеет вид [14]

$\epsilon_{\nu n}^{\mathrm{FL}}=-\frac{1}{2} \operatorname{Re}\left(\psi^{\prime \prime}\left(1+i \nu+\frac{|n|}{2}\right)-\frac{2 i \nu \psi^{\prime}(1+i \nu+|n| / 2)}{\nu^{2}+n^{2} / 4}\right)-\zeta(2) E_{\nu n}-\frac{1}{4} \frac{|n|\left(\nu^{2}-n^{2} / 4\right)}{\left(\nu^{2}+n^{2} / 4\right)^{3}}$.

Первые поправки к ядру уравнения БКП также были вычислены с применением подхода эффективного действия в работах [15].

\section{3. ПОМЕРОН И ГРАВИТОН \\ В $N=4$ СУПЕРСИММЕТРИЧНОЙ ТЕОРИИ}

В соответствии с соотношением АдС/КТП [16]-[18] померон БФКЛ в конформной $N=4$ суперсимметричной калибровочной теории связан с гравитоном в дуальном десятимерном пространстве анти-де Ситтера [19]. Собственное значение ядра БФКЛ в диффузионном приближении

$$
j=2-\Delta-\Delta \nu^{2}, \quad \gamma=1+\frac{j-2}{2}+i \nu
$$

удовлетворяет закону сохранения энергии-импульса $\left.\gamma(j)\right|_{j=2}=0$ и зависит от параметра $\Delta$, который можно вычислить по теории возмущений [2]. С другой стороны, можно использовать известное соответствие АдС/КТП [17], [18] и представить собственное значение ядра БФКЛ в диффузионном приближении в виде линейной траектории Редже гравитона в десятимерном пространстве анти-де Ситтера:

$$
j=2+\frac{\alpha^{\prime}}{2} t, \quad t=\frac{E^{2}}{R^{2}}, \quad \alpha^{\prime}=\frac{R^{2}}{2} \Delta .
$$

Применяя результат Губсера, Клебанова и Полякова об асимптотическом поведении аномальных размерностей операторов твиста 2 для $\gamma=1-\sqrt{1+(j-2) / \Delta}$, можно 
получить ведущую поправку к интерсепту гравитона $j=2$ при больших значениях константы связи [20], [21]:

$$
\Delta=\frac{1}{\sqrt{\lambda}}, \quad \lambda=g^{2} N_{\mathrm{c}}
$$

Заметим, что наклон графика аномальной размерности при $j=2$ тесно связан с интерсептом померона. Он был вычислен с точностью до пятого порядка теории возмущений [20], [22]. Недавно величина $\gamma^{\prime}(2)$ была получена во всех порядках [23]:

$$
\gamma^{\prime}(2)=-\frac{\lambda}{24}+\frac{1}{2} \frac{\lambda^{2}}{24^{2}}-\frac{2}{5} \frac{\lambda^{3}}{24^{2}}+\frac{7}{20} \frac{\lambda^{4}}{24^{4}}-\frac{11}{35} \frac{\lambda^{5}}{24^{5}}+\cdots=-\frac{\sqrt{\lambda}}{4} \frac{I_{3}(\sqrt{\lambda})}{I_{2}(\sqrt{\lambda})} .
$$

Эта формула выведена с применением асимптотического анзаца Бете для аномальных размерностей в $N=4$ суперсимметричной теории [23]. Появление интегрируемой спиновой цепочки Гейзенберга в уравнениях для аномальных размерностей квазипартонных операторов [24] в ВЛП в $N=4$ суперсимметричной теории обнаружено впервые в работе [25]. Дуальность между помероном БФКЛ и гравитоном в рамках соотношения АдС/КТП в $N=4$ суперсимметричной теории означает, что померонную теорию Грибова следует переформулировать в терминах общековариантной теории реджезованных гравитонов. Именно поэтому ниже мы исследуем высокоэнергетическое эффективное действие, локальное по гравитонным быстротам.

\section{4. ВЫСОКОЭНЕРГЕТИЧЕСКИЕ АМПЛИТУДЫ В ГРАВИТАЦИИ}

Амплитуда множественного рождения гравитонов при высокоэнергетических гравитон-гравитонных соударениях была получена в работе [26]:

$$
A_{2 \rightarrow n}=-s^{2} \Gamma_{\mu \nu}^{\mu^{\prime} \nu^{\prime}} \frac{s_{1}^{\omega\left(q_{1}^{2}\right)}}{q_{1}^{2}} \Gamma_{\rho_{1} \sigma_{1}} \frac{s_{2}^{\omega\left(q_{2}^{2}\right)}}{q_{2}^{2}} \Gamma_{\rho_{2} \sigma_{2}} \ldots \Gamma_{\rho \sigma}^{\rho^{\prime} \sigma^{\prime}} .
$$

Эффективная вершина взаимодействия гравитон-гравитон-реджезованный гравитон

$$
\Gamma_{\mu \nu}^{\mu^{\prime} \nu^{\prime}}=\frac{\kappa}{4}\left(\Gamma_{\mu \mu^{\prime}} \Gamma_{\nu \nu^{\prime}}+\Gamma_{\mu \nu^{\prime}} \Gamma_{\nu \mu^{\prime}}\right)
$$

может быть выражена в терминах вершины взаимодействия глюон-глюон-реджезованный глюон

$$
\Gamma_{\mu \mu^{\prime}}=-\delta_{\mu \mu^{\prime}}+\frac{p_{\mu^{\prime}}^{A} p_{\mu}^{B}+p_{\mu}^{A^{\prime}} p_{\mu^{\prime}}^{B}}{p^{A} p^{B}}+\frac{q^{2}}{2} \frac{p_{\mu}^{B} p_{\mu^{\prime}}^{B}}{\left(p^{A} p^{B}\right)^{2}} .
$$

Похожим образом вершина взаимодействия реджеон-реджеон-гравитон

$$
\Gamma_{\rho \sigma}=\frac{\kappa}{4}\left(C_{\rho} C_{\sigma}-N_{\rho} N_{\sigma}\right), \quad N=\sqrt{q_{1}^{2} q_{2}^{2}}\left(\frac{p^{A}}{k p^{A}}-\frac{p^{B}}{k p^{B}}\right)
$$

содержит наряду с вершиной $N$ сопровождающего излучения КЭД также вершину эффективного взаимодействия реджеон-реджеон-глюон в КХД:

$$
C=-q_{1}^{\perp}-q_{2}^{\perp}+p^{A}\left(\frac{q_{1}^{2}}{k p^{A}}+\frac{k p^{B}}{p^{A} p^{B}}\right)-p^{B}\left(\frac{q_{2}^{2}}{k p^{B}}+\frac{k p^{A}}{p^{A} p^{B}}\right) .
$$


Траектория гравитона задается формулой [26]

$$
\omega\left(q^{2}\right)=\frac{\alpha}{\pi} \int d^{2} k \frac{q^{2}}{k^{2}(q-k)^{2}}\left((k, q-k)^{2}\left(\frac{1}{k^{2}}+\frac{1}{(q-k)^{2}}\right)-q^{2}+\frac{N}{2}(k, q-k)\right),
$$

где $\kappa$ - постоянная Эйнштейна, $\alpha=\kappa^{2} / 8 \pi^{2}$, а $N$ - число гравитино, возникающих в $N$-расширенной теории супергравитации. Действие гравитино хорошо известно:

$$
S_{3 / 2}=-\frac{1}{2} \epsilon^{\mu \nu \rho \sigma} \int d^{4} x \sum_{r=1}^{N} \bar{\psi}_{\mu}^{r} \gamma_{5} \gamma_{\nu} \partial_{\rho} \psi_{\sigma}^{r}
$$

Траектория гравитона содержит как инфракрасные, так и ультрафиолетовые расходимости:

$$
\omega\left(q^{2}\right)=-\alpha|q|^{2}\left(\ln \frac{|q|^{2}}{\lambda^{2}}+\frac{N-4}{2} \ln \frac{|\Lambda|^{2}}{|q|^{2}}\right) .
$$

Инфракрасная расходимость имеет универсальный характер, в то время как расходимость траектории в ультрафиолетовой области зависит от $N$ и отсутствует в $N=4$ теории супергравитации.

Чтобы вычислить траекторию гравитона и гравитонные взаимодействия в старших порядках теории возмущений, можно построить эффективное действие, локальное по быстротам реджезованных и обычных гравитонов

$$
y=\frac{1}{2} \ln \frac{\epsilon_{k}+|k|}{\epsilon_{k}-|k|}, \quad\left|y-y_{0}\right|<\eta, \quad \eta \ll \ln s .
$$

Поля $A^{++}$и $A^{--}$, описывающие рождение и уничтожение реджезованных гравитонов, оказываются общековариантными и удовлетворяют дополнительным кинематическим связям

$$
\delta A^{++}(x)=\delta A^{--}(x)=0, \quad \partial_{+} A^{++}(x)=\partial_{-} A^{--}(x)=0 .
$$

В терминах этих полей эффективное действие принимает вид [27]

$$
S=-\frac{1}{2 \kappa} \int d^{4} x\left(\sqrt{-g} R+\frac{1}{2}\left(\partial_{+} j^{-} \partial_{\mu}^{2} A^{++}+\partial_{-} j^{+} \partial_{\mu}^{2} A^{--}\right)\right) .
$$

Токи $j^{ \pm}=2 x^{ \pm}-\omega^{ \pm}$удовлетворяют уравнению Гамильтона-Якоби

$$
g^{\mu \nu} \partial_{\mu} \omega^{ \pm} \partial_{\nu} \omega^{ \pm}=0, \quad \partial_{ \pm} j^{\mp}=h_{ \pm \pm}-\left(h_{\rho \pm}-\frac{1}{2} \frac{\partial_{\rho}}{\partial_{ \pm}} h_{ \pm \pm}\right)^{2}+\cdots
$$

которое можно решить в рамках теории возмущений. Поправки в случае гравитационного поля типа ударной волны с метрикой Айхельбурга-Сексля можно вычислить в явном виде [27]. 


\section{5. ДВАЖДЫ-ЛОГАРИФМИЧЕСКОЕ ПРИБЛИЖЕНИЕ АМПЛИТУДЫ В ГРАВИТАЦИИ}

Для вычисления амплитуды рассеяния гравитона на гравитоне в дважды-логарифмическом приближении удобно использовать преобразование Меллина

$$
A(s, t)=A_{\mathrm{Born}} s^{-\alpha|q|^{2} \ln \left(|q|^{2} / \lambda^{2}\right)} \int_{a-i \infty}^{a+i \infty} \frac{d \omega}{2 \pi i \omega}\left(\frac{s}{|q|^{2}}\right)^{\omega} f_{\omega}, \quad \alpha=\frac{\kappa^{2}}{8 \pi^{2}} .
$$

В общем случае $N$-расширенной супергравитации парциальная волна в $t$-канале рассеяния удовлетворяет уравнению эволюции в инфракрасной области [28]

$$
f_{\omega}=1+b \frac{d}{d \omega} \frac{f_{\omega}}{\omega}-b \frac{N-6}{2} \frac{f_{\omega}^{2}}{\omega^{2}}, \quad b=\alpha|q|^{2},
$$

которое напоминает уравнения, полученные ранее в КЭД и КХД [29]. Идея, позволяющая получить эти уравнения, похожа на подход ренормализационной группы. А именно, в фейнмановских диаграммах для амплитуды рассеяния выбираются диаграммы, соответствующие виртуальным гравитонам с минимальными значениями $k_{\perp}$ поперечных импульсов, таким образом, чтобы остающиеся при этом части фейнмановских вкладов могли быть снова выражены в терминах этих амплитуд, но уже с другим параметром (порядка $k_{\perp}^{2}$ ) инфракрасного обрезания. Можно найти явное решение этого эволюционного уравнения в терминах функций параболического цилиндра:

$$
\frac{f_{\omega}^{(N)}}{\omega}=\frac{2}{6-N} \frac{1}{\sqrt{b}} \frac{d}{d x} \ln d^{(N)}(x), \quad d^{(N)}(x)=e^{x^{2} / 4} D_{(6-N) / 2}(x), \quad x=\frac{\omega}{\sqrt{b}} .
$$

Разложение амплитуд рассеяния в ряд теории возмущений по параметру двойного логарифма $\xi=\alpha|t| \ln ^{2}\left(s /|q|^{2}\right)$ имеет вид

$$
A(s, t)=A_{\mathrm{Born}} s^{-\alpha|q|^{2} \ln \left(|q|^{2} / \lambda^{2}\right)} \Phi(\xi),
$$

где

$$
\Phi(\xi)=1-\frac{N-4}{2} \frac{\xi}{2}+\frac{(N-4)(N-3)}{2} \frac{\xi^{2}}{4 !}+\cdots
$$

и находится в согласии с точными двухпетлевыми результатами. Оказывается, что для больших $\xi$ асимптотическое поведение амплитуды зависит от числа гравитино так, что при $N>5$ амплитуда рассеяния быстро убывает по сравнению с борновским вкладом.

В дважды-логарифмическом приближении можно также вычислить амплитуды множественного рождения гравитонов

$$
s \gg s_{i}=\left(k_{i-1}+k_{i}\right)^{2} \gg\left|k_{r}^{\perp}\right|^{2}, \quad \prod_{r=1}^{n+1} s_{r}=s \prod_{r=1}^{n}\left|k_{r}\right|^{2} .
$$

Например, амплитуда рождения гравитона в этом приближении имеет вид

$$
A_{2 \rightarrow 3}^{(N)}=A_{2 \rightarrow 3}^{\text {Born }} s_{1}^{-\alpha\left|q_{1}\right|^{2} \ln \left(\left|q_{1}\right|^{2} / \mu^{2}\right)} s_{2}^{-\alpha\left|q_{2}\right|^{2} \ln \left(\left|q_{2}\right|^{2} / \mu^{2}\right)} r^{(N)}\left(\rho_{1}, \rho_{2}\right), \quad \rho_{r}=\sqrt{\alpha} \frac{\ln s_{r}}{\left|q_{r}\right|^{2}},
$$


где

$$
\begin{aligned}
r^{(N)}= & \int_{-i \infty}^{+i \infty} \frac{d x_{1} d x_{2}}{(2 \pi i)^{2}} e^{x_{1} \rho_{1}+x_{2} \rho_{2}} \times \\
& \times \frac{d^{(N)}\left(\sqrt{\left|q_{2}\right| /\left|q_{1}\right|} x_{1}\right) d^{(N)}\left(\sqrt{\left|q_{1}\right| /\left|q_{2}\right|} x_{2}\right)}{d^{(N)}\left(x_{1}\right) d^{(N)}\left(x_{2}\right)} \phi^{(N)}\left(x_{1}, x_{2}\right), \\
\phi^{(N)}\left(x_{1}, x_{2}\right)= & \frac{2}{6-N}\left(\frac{\left(d^{(N)}\left(\sqrt{\left|q_{2}\right| /\left|q_{1}\right|} x_{1}\right)\right)^{\prime}}{d^{(N)}\left(\sqrt{\left|q_{2}\right| /\left|q_{1}\right|} x_{1}\right)}-\frac{\left(d^{(N)}\left(\sqrt{\left|q_{1}\right| /\left|q_{2}\right|} x_{2}\right)\right)^{\prime}}{d^{(N)}\left(\sqrt{\left|q_{1}\right| /\left|q_{2}\right|} x_{1}\right)}\right) \times \\
& \times \frac{1}{\sqrt{\left|q_{1}\right| /\left|q_{2}\right|} x_{2}-\sqrt{\left|q_{2}\right| /\left|q_{1}\right|} x_{1}} .
\end{aligned}
$$

Можно также найти поправки типа двойных логарифмов к выражениям для амплитуд рассеяния в эйкональном приближении с помощью модификации эйкональной фазы $\delta(\vec{\rho}, \ln s)$ в соответствии с вышеприведенными асимптотиками двойных логарифмических функций:

$$
\begin{aligned}
A_{\mathrm{DL}}(s, t) & =-2 i s s^{-\alpha|q|^{2} \ln \left(|q|^{2} / \mu^{2}\right)} \int d^{2} \rho e^{i \vec{q} \vec{\rho}}\left(e^{i \delta_{\mathrm{DL}}(\vec{\rho}, \ln s)}-1\right), \\
\delta_{\mathrm{DL}}(\vec{\rho}, \ln s) & =\frac{s}{6-N} \frac{\kappa^{2}}{(2 \pi)^{2}} \int \frac{d^{2} q}{|q|^{2}} e^{-i \vec{q} \vec{\rho}} \int_{-i \infty}^{i \infty} \frac{d x}{2 \pi i} s^{\sqrt{\alpha}|q| x} \frac{\left(d^{(N)}(x)\right)^{\prime}}{d^{(N)}(x)}= \\
& =\frac{s}{2} \frac{\kappa^{2}}{(2 \pi)^{2}} \int \frac{d^{2} q}{|q|^{2}} e^{-i \vec{q} \vec{\rho}} \Phi(\xi),
\end{aligned}
$$

где функция $\Phi(\xi)$ задана в $(10)$, а $d^{(N)}(x)$ выражается в терминах функции параболического цилиндра (см. формулы (9)). Разложение для этой амплитуды имеет вид

$$
A_{\mathrm{DL}}(s, t)=-2 i s s^{-\alpha|q|^{2} \ln \left(|q|^{2} / \mu^{2}\right)}(2 \pi)^{2} \sum_{n=0}^{\infty} \frac{(s \alpha i)^{n}}{n !} \int \prod_{r=1}^{n} \frac{d^{2} q_{r}}{\left|q_{r}\right|^{2}} \Phi\left(\xi_{r}\right) \delta^{2}\left(q-\sum_{l=1}^{n} q_{l}\right),
$$

где $\left|q_{r}\right|^{2}<s$. Это предсказание можно частично проверить на уровне двух петель: для члена, пропорционального $\kappa^{2} s^{3} \alpha^{2}$, получим

$$
\left.A_{4}^{N}\right|_{N=8}=\frac{\kappa^{2} s^{2}}{|q|^{2}}(-i \pi s) \alpha^{2}|q|^{2} \frac{\ln ^{3}\left(s /|q|^{2}\right)}{3}
$$

что согласуется с известным двухпетлевым результатом.

\section{6. ОБСУЖДЕНИЕ}

Мы показали, что померон в КХД представляет собой объект, составленный из реджезованных глюонов. Чтобы построить траектории Редже для глюона и взаимодействия реджеонов, можно применить подход эффективного действия. При больших $N_{\text {c }}$ уравнение БКП для связанных состояний глюонов в цветовом синглетном и октетном представлениях оказывается интегрируемым. В калибровочной $N=4$ теории с расширенной суперсимметрией померон дуален гравитону в 
десятимерном пространстве анти-де Ситтера. В результате теория померона Грибова в этой модели должна быть основана на теории взаимодействующих реджезованных гравитонов. Траектории и вершины гравитонов в теории возмущений можно вычислить с помощью эффективного действия, локального по быстротам частиц. В низшем порядке траектория гравитона содержит логарифмическую расходимость в ультрафиолетовой области, поэтому в гравитации возникают поправки типа двойных логарифмов. Показано, что в дважды-логарифмическом приближении суперсимметрия приводит к более быстрому убыванию амплитуды рассеяния при высоких энергиях по сравнению с борновскими выражениями.

\section{Список литературы}

[1] Л. Н. Липатов, ЯФ, 23 (1976), 642-656; V.S. Fadin, E. A. Kuraev, L. N. Lipatov, Phys. Lett. B, 60:1 (1975), 50-52; Е. А. Кураев, Л.Н. Липатов, В. С. Фадин, ЖЭТФ, $\mathbf{7 1}$ (1976), 840-855; 72 (1977), 377-389; Я. Я. Балицкий, Л. Н. Липатов, ЯФ, 28 (1978), $1597-1611$.

[2] V.S. Fadin, L. N. Lipatov, Phys. Lett. B, 429:1-2 (1998), 127-134, arXiv: hep-ph/9802290; M. Ciafaloni, G. Camici, Phys. Lett. B, 430:3-4 (1998), 349-354, arXiv: hep-ph/9803389;

A. V. Kotikov, L. N. Lipatov, Nucl. Phys. B, 582:1-3, 19-43.

[3] L. N. Lipatov, Phys. Lett. B, 309:3-4 (1993), 394-396.

[4] Л.Н.Липатов, ЖЭТФ, 90:5 (1986), 1536-1552.

[5] L. N. Lipatov, Phys. Lett. B, 251:2 (1990), 284-287; "Pomeron in quantum chromodynamics", Perturbative Quantum Chromodynamics, Advanced Series on Directions in High Energy Physics, 5, ed. A. H. Mueller, World Sci., Singapore, 1989, 411-489.

[6] A. A. Belavin, A. M. Polyakov, A. B. Zamolodchikov, Nucl. Phys. B, 241:2 (1984), 333-380.

[7] L. N. Lipatov, Nucl. Phys. B, 548:1-3 (1999), 328-362, arXiv: hep-ph/9812336.

[8] Л.Н. Липатов, Писъма в ЖЭТФ, 59 (1994), 571-574, arXiv: hep-th/9311037.

[9] Р. Бакстер, Точно решаемые модели в статистической механике, Мир, М., 1985.

[10] L.D. Faddeev, G. P. Korchemsky, Phys. Lett. B, 342:1-4 (1995), 311-322, arXiv: hep-th/9404173.

[11] L. N. Lipatov, Nucl. Phys. B, 452:1-2 (1995), 369-397; Phys. Rept., 286 (1997), 131-198.

[12] E. N. Antonov, I. O. Cherednikov, E. A. Kuraev, L. N. Lipatov, Nucl. Phys. B, 721:1-3 (2005), 111-135, arXiv: hep-ph/0411185.

[13] Z. Bern, L. J. Dixon, V. A. Smirnov, Phys. Rev. D, 72:8 (2005), 085001, 27 pp., arXiv: hep-th/0505205.

[14] V.S. Fadin, L. N. Lipatov, Phys. Lett. B, 706:4-5 (2012), 470-476.

[15] J. Bartels, L. N. Lipatov, G. P. Vacca, Ward identities for amplitudes with reggeized gluons, arXiv: 1205.2530; J. Bartels, V.S. Fadin, L. N. Lipatov, G. P. Vacca, NLO corrections to the kernel of the BKP-equations, arXiv: 1210.0797.

[16] J. Maldacena, Adv. Theor. Math. Phys., 2 (1998), 231-252.

[17] S. S. Gubser, I. R. Klebanov, A. M. Polyakov, Phys. Lett. B, 428:1-2 (1998), 105-114, arXiv: hep-th/9802109.

[18] E. Witten, Adv. Theor. Math. Phys., 2:2 (1998), 253-291, arXiv: hep-th/9802150.

[19] J. Polchinski, M. J. Strassler, JHEP, 05 (2003), 012, 37 pp., arXiv: hep-th/0209211.

[20] A. V. Kotikov, L. N. Lipatov, A. V. Onishchenko, V. N. Velizhanin, Phys. Lett. B, 595:1-4 (2004), 521-529, arXiv: hep-th/0404092; Erratum, 632:5-6 (2006), 754-756.

[21] R. C. Brower, J. Polchinski, M. J. Strassler, C. I. Tan, JHEP, 12 (2007), 005, 62 pp., arXiv: hep-th/0603115.

[22] T. Łukowski, A. Rej, V.N. Velizhanin, Nucl. Phys. B, 831:1-2 (2010), 105-132, arXiv: 0912.1624 . 
[23] B. Basso, An exact slope for AdS/CFT, arXiv: 1109.3154.

[24] A. P. Bukhvostov, G. V. Frolov, L. N. Lipatov, E. A. Kuraev, Nucl. Phys. B, 258 (1985), 601-646.

[25] L. N. Lipatov, "Evolution equations in QCD", Perspectives in Hadronic Physics, Proceedings of the Conference ICTP (Trieste, Italy, 12-16 May, 1997), eds. S. Boffi, M. M. Giannini, C. Ciofi Degli Atti, World Sci., Singapore, 1997, 413-427.

[26] L. N. Lipatov, Phys. Lett. B, 116:6 (1982), 411-413; Л. Н. Липатов, ЖЖЭФФ, 82:4 (1982), 991-1006.

[27] L. N. Lipatov, Effective action for the Regge processes in gravity, arXiv: 1105.3127.

[28] J. Bartels, L. N. Lipatov, A. Sabio Vera, Double-logarithms in Einstein-Hilbert gravity and supergravity, arXiv: 1208.3423.

[29] R. Kirschner, L. N. Lipatov, Nucl. Phys. B, 213:1 (1983), 122-148. 